\title{
Cognitive abilities and life experience in everyday planning in adolescents with intellectual disabilities: Support for the difference model
}

Lisa Palmqvist, Henrik Danielsson, Arne Jönsson and Jerker Rönnberg

The self-archived postprint version of this journal article is available at Linköping University Institutional Repository (DiVA):

http://urn.kb.se/resolve?urn=urn:nbn:se:liu:diva-163409

N.B.: When citing this work, cite the original publication.

Palmqvist, L., Danielsson, H., Jönsson, A., Rönnberg, J., (2020), Cognitive abilities and life experience in everyday planning in adolescents with intellectual disabilities: Support for the difference model, Journal of Intellectual Disability Research. https://doi.org/10.1111/jir.12710

Original publication available at:

https://doi.org/10.1111/jir.12710

Copyright: Wiley (12 months)

http://eu.wiley.com/WileyCDA/ 
Cognitive abilities and life experience in everyday planning in adolescents with intellectual disabilities: Support for the difference model

Lisa Palmqvist ${ }^{1,2}$, Henrik Danielsson ${ }^{1,2}$, Arne Jönsson ${ }^{3}, \&$ Jerker Rönnberg ${ }^{1,2}$

${ }^{1}$ Department of Behavioural Sciences and Learning, Linköping University

${ }^{2}$ The Swedish Institute of Disability Research

${ }^{3}$ Department of Computer and Information Science, Linköping University 
Running head: Planning and Intellectual Disabilities: Support for the difference model

\section{Author note}

Correspondence concerning this article should be addressed to Lisa Palmqvist, IBL, Linköping University, 58183 Linköping, Sweden. E-mail: lisa.palmqvist@liu.se 
Running head: Planning and Intellectual Disabilities: Support for the difference model

\begin{abstract}
Background: The literature on planning ability in individuals with intellectual disability (ID) provides no clarity on whether their ability matches their mental age (MA) or not. Perhaps can planning experience explain the mixed results. The current study investigated to what extent cognitive abilities and life experience can explain everyday planning ability in individuals with ID and to what extent results from everyday planning tasks support the developmental or the difference model of ID. Method: Planning tests, cognitive ability tasks and self-rated life experience were administered to 71 adolescents with ID and 62 children with a typical development matched on mental age (MA). Results: Adolescents with ID exhibited planning ability according to their MA. Regression analyses showed that the predictors of planning differed between the groups. The cognitive measures could predict planning in both groups, but life experience only contributed positively to planning in the MA group, whereas chronological age was negatively correlated with successful planning in the ID group. Conclusions and discussion: The results support the difference model of ID. When matched on MA, the individuals with ID will solve the planning task in a qualitatively different manner. Additionally, the participants with ID could not utilise their life experience when solving the planning task, contrary to the MA group. Practitioners should be aware that individuals with ID might need more everyday planning training throughout adolescence. To support adolescents with ID, practitioners may focus on supporting the individual's cognitive abilities rather than relying on their prior knowledge.
\end{abstract}

Keywords: Intellectual disability, everyday planning, cognitive abilities, errand task, difference model. 
Running head: Planning and Intellectual Disabilities: Support for the difference model

Cognitive abilities and life experience in everyday planning in adolescents with intellectual disabilities: Support for the difference model

\section{Introduction}

Planning is central to everyday life. It includes both short-term planning, such as morning scheduling, and long-term planning, like career planning. When planning, the individual formulates, develops, and organises sub-goals to achieve a broader goal. Plan formulation involves both life experience and cognitive abilities. Individuals with intellectual disabilities (ID) are known to have planning difficulties (e.g. Danielsson, Henry, Messer, \& Rönnberg, 2012; Lanfranchi, Jerman, Dal Pont, Alberti, \& Vianello, 2010). Research is scarce on if both life experience and cognitive abilities are involved in everyday planning in individuals with ID, or to which extent individuals with ID perform on par, or qualitatively different from MA-matched individuals with a typical development (TD).

\section{Planning}

Planning is the construction of an appropriate course of actions to reach a goal (e.g. B. Hayes-Roth \& Hayes-Roth, 1979; Miyake et al., 2000; Pennington \& Ozonoff, 1996; Scholnick \& Friedman, 1993). When formulating a plan, the sub-tasks and their temporal order must be identified (B. Hayes-Roth \& Hayes-Roth, 1979; Levine et al., 2000). For an everyday planning task, planners will use prior knowledge of a similar situation, together with cognitive resources (S. L. Friedman \& Scholnick, 2014; Scholnick \& Friedman, 1993). Thus, planning is defined as requiring cognitive functions as well as life experience (B. Hayes-Roth \& Hayes-Roth, 1979; Scholnick \& Friedman, 1993). 
Running head: Planning and Intellectual Disabilities: Support for the difference model

\section{Cognitive processes associated with planning}

Executive functions, a set of higher-order, top-down cognitive abilities, are often considered a core element in goal-directed behaviours such as planning (Diamond, 2013; Miyake et al., 2000; Pennington \& Ozonoff, 1996). When generating, sequencing, and monitoring the formulation of a plan, the planner needs to engage their working memory capacity (WM) to manipulate the information in their mind. Visuo-spatial WM has been found important for planning since planning includes a spatial-temporal component (D’Antuono et al., 2017; Numminen, Lehto, \& Ruoppila, 2001). Cognitive flexibility influences planning as the planner needs to switch between different sub-goals as the plan progresses (Brookshire, Levin, Song, \& Zhang, 2004; Pellicano, 2010). Alongside with executive functions, inductive reasoning and verbal fluency have been associated with planning ability (Brookshire et al., 2004; D'Antuono et al., 2017; Unterrainer et al., 2004).

\section{Life experience and planning}

The planner's past knowledge helps in identifying relevant actions to reach a goal (Scholnick \& Friedman, 1993). Kliegel, Martin, McDaniel, and Phillips (2007) compared old to young adults on familiar and novel planning tasks. They found that in familiar situations the groups performed on par, whereas the old adults were outperformed on novel tasks. Kliegel et al. (2007) concluded that older adults compensated for their cognitive decline by utilising taskrelevant knowledge. On the same note, it is possible that individuals with ID, adopt similar strategies and utilise life experience to compensate for lower cognitive abilities. If so, does longer life experience help them plan better in everyday situations compared to younger MA-matched peers? 
Running head: Planning and Intellectual Disabilities: Support for the difference model

\section{Planning and intellectual disabilities}

It has been debated whether ID can best be explained in terms of a developmental delay, or if the development of individuals with ID is both qualitatively and quantitatively different from individuals with a TD (Bennett-Gates \& Zigler, 1998). The developmental model distinguishes between cultural-familial and organic ID, stating that the difference model is only valid for cognitive traits in organic ID. Cultural-familial ID is thought to follow the same developmental path as the normal population, but with at a slower rate. Traits in an organic ID, on the other hand, would be manifested by both a lower performance and different resources needed to complete the tasks. This view contradicts the empirical evidence, showing support for the difference model also in individuals with no known biological deficit (Danielsson et al., 2012; Schuchardt, Gebhardt, \& Mähler, 2010).

The literature provides no clear picture of planning ability for individuals with ID. For instance, literature have found individuals with ID to perform below both peers matched on chronological age (CA) and on mental age (MA; Danielsson et al., 2012; Lanfranchi et al., 2010; Rowe, Lavender, \& Turk, 2006), while Pennington, Moon, Edgin, Stedron, and Nadel (2003) and Numminen et al. (2001) found individuals with ID performed according to their MA. A study by Danielsson, Henry, Rönnberg, and Nilsson (2010) even found that individuals with ID performed on par with CA matched peers. When the findings show such disparity, unaccounted moderators might explain some of the variances in results. Difficulties with testing the ID population could be one explanation. Due to the heterogeneity of the population, there might be inherent differences stemming from having an ID. The cognitive tests are often developed for a TD population and the population with ID might have difficulties with understanding the tests 
Running head: Planning and Intellectual Disabilities: Support for the difference model

(Masson, Dagnan, \& Evans, 2010; Willner, Bailey, Parry, \& Dymond, 2010). Researchers should carefully choose tests that are adapted for the population to ensure that the participant understands the task. Another unaccounted moderator could be the participant's life experience. Therefore, we included a planning task that simulated an everyday context and collected proxy estimates of the participants' life experience.

\section{Assessing everyday planning}

Few tasks investigate planning that simulates everyday behaviour (e.g. Ardila, 2008; Burgess, Alderman, Evans, Emslie, \& Wilson, 1998; Burgess et al., 2006; Jurado \& Rosselli, 2007; Simon, 1975). Even fewer tasks have been developed for individuals with ID. Errand tasks, where the participant is asked to plan a sequence of errands, has been developed to measure everyday planning (Chalmers \& Lawrence, 1993; e.g. Kliegel et al., 2007; Shallice \& Burgess, 1991). The test can be done either by using pen-and-paper or by asking the participant to run actual everyday errands. The test is thus advantageous for investigating the influence of both cognitive abilities and life experience compared to tasks without everyday context (e.g. Tower of London; Shallice, 1982). Several versions exist for populations with cognitive difficulties (e.g. Emslie, Wilson, Burden, Nimmo-Smith, \& Wilson, 2003; Shallice \& Burgess, 1991; Steverson, Adlam, \& Langdon, 2017). Perhaps, the most well-known errand task is the Multiple Errand Task (MET; Shallice \& Burgess, 1991), where the planner is asked to run a set of errands in a shopping mall while following a set of rules. Two additional errand tasks are the Children's Kitchen Task Assessment (CKTA; Chevignard, Catroppa, Galvin, \& Anderson, 2010), and the Zoo map test (BADS-C; Emslie et al., 2003). 
Running head: Planning and Intellectual Disabilities: Support for the difference model

\section{Current study and research questions}

To conclude, planning skills are based on both the planner's cognitive abilities and prior experiences. More specifically, life experience, inductive reasoning, visuo-spatial WM, cognitive flexibility, and verbal fluency have been associated with performance on planning tests. Research on planning in individuals with ID show mixed results as to whether they perform according to the developmental or difference model of ID. We propose that the differences in the literature may be moderated by the participants' life experience. This study examined how cognitive abilities and life experience relate to planning in an everyday context in adolescents with ID compared to MA matched children with a TD. Thus, allowing a comparison of the extent to which planning abilities can be attributed to cognitive abilities or life experience. The study also evaluated these results to the developmental and the difference model of ID. Accordingly, our research questions were:

1. To what extent can cognitive abilities and life experience explain performance in everyday planning in individuals with intellectual disabilities?

2. Which results from everyday planning are more consistent with the development delay model and which are more consistent with the difference model of intellectual disability?

\section{Methods}

\section{Participants}

The participants in the present study are part of a larger intervention project that aimed to improve everyday planning. The data from the present study come from before the intervention started. For the study, we recruited 143 participants ( 80 adolescents with ID and 63 younger children with a TD) from schools in southern Sweden. The participants in the ID group were 
Running head: Planning and Intellectual Disabilities: Support for the difference model

recruited from upper secondary schools for children with special needs. In Sweden, the enrolment criterion is to have a mild to moderate ID diagnosis, which is made after a thorough assessment by a licensed psychologist. The comparison group were recruited from primary schools.

To evaluate the research questions, the groups were matched on MA (on raw scores from Raven's coloured progressive matrices; RCPM; Raven, 2003). Facon, Magis, and Belmont (2011) suggested groups be equated on both mean and variance, with a suggested $\alpha$-level of $p>$ 0.05. The ID group had lower MA than the comparison group and seven participants (six with ID) with scores below 15 (corresponding to a mental age of 6 years) were excluded. After removing three more ID participants with low scores, the groups did neither differ in mean, $t(130.46)=0.59, p=.555$, nor variance, Levene's test: $F(1,131)=0.61, p=.434$. The matched groups consisted of 133 participants: $n_{I D}=71(65 \%$ girls, $\mathrm{CA}=18.10(1.30)$ years, MA $=8.39(1.40)$ years and $n_{M A}=62(47 \%$ girls, $\mathrm{CA}=7.84(0.73)$ years, $\mathrm{MA}=8.55(1.25)$ years.

Background data on diagnoses were collected using parental surveys. Diagnoses in the ID group is presented in Table 1, descriptive statistics on chronological age and RCPM (denoted as inductive reasoning) in Table 3. No neuro-developmental disorders were reported in the MA group, but data was missing for 14 participants. However, the teachers reported that none of the included students from the MA group had special educational needs.

\section{Insert Table 1 about here}

Participation was voluntary. The participants were recruited after getting consent from the school's principal, thereafter the participants' caregivers (and participants over 18 years) were 
Running head: Planning and Intellectual Disabilities: Support for the difference model

given an information letter about the study, that all data would be treated confidentially, and analysed at group-level. All caregivers signed an informed consent before participation. The participants gave oral consent at the beginning of the test session. The study was reviewed and approved by the Regional Ethical Review Board in Linköping, Sweden (2015/119-31).

\section{Materials}

Three areas were investigated: 1) everyday planning, 2) cognitive abilities, and 3) life experience. Everyday planning was assessed using four planning tests: Order-your-task (OYT), Multiple errand task (MET; Shallice \& Burgess, 1991; Steverson et al., 2017), Children's Kitchen Task Assessment (CKTA; Chevignard et al., 2010), and the Zoo map task (Emslie et al., 2003). The planning tests varied in complexity and experiential areas of everyday planning. The scores were later combined into one measure to capture a broad picture of everyday planning. To improve the reliability of the planning scores, mean scores of two similar versions of the MET, CKTA, and Zoo map, were calculated. Instructions for the planning tests were given verbally, with pictures (from the library WidgitGo $\mathbb{C}$ ) and written. The included cognitive tests assessed: Executively loaded visuo-spatial WM capacity (Corsi span; Mueller \& Piper, 2014), Inductive reasoning (RCPM; Raven, 2003), Cognitive flexibility (Playing Cards from BADS-C; Emslie et al., 2003), and Verbal fluency (semantic category fluency; Baldo, Shimamura, Delis, Kramer, \& Kaplan, 2001). Proxies for life experience was the participant's CA and ratings of activity experience (Arvidsson \& Granlund, 2018). All tests but the verbal fluency test and the ratings of activity experience were non-verbal. Reliability scores using Cronbach's $\alpha$ were calculated for the separate planning tests: Zoo map ( $\alpha=0.70)$, CKTA ( $\alpha=0.42)$, MET ( $\alpha=0.66)$, and OYT ( $\alpha$ 
Running head: Planning and Intellectual Disabilities: Support for the difference model

$=0.63)$, the reliability was slightly higher in the ID group compared to the MA group. A detailed test description is presented in Table 2.

\section{Insert Table 2 about here}

\section{Procedure}

All participants were tested individually in a separate room in their school environment. The testing was either done in one session or split into two, depending on participants' or schools' preference. No test had a time limit. Therefore, assessment duration differed between participants, ranging from one to two hours.

\section{Data analysis and design}

All statistical analyses were calculated using R 3.4.3, R Studio version 1.1.456. The following r-packages were used: Wickham, François, Henry, and Müller (2019), Todorov and Filzmoser (2009), van Buuren and Groothuis-Oudshoorn (2011), Fox and Weisberg (2011), Aust and Barth (2018) and Revelle (2018). The $\alpha$-value was set to 0.05 .

\section{Everyday planning, cognitive abilities, and life experience A Principal} Component Analysis (PCA) was performed to combine the planning variables into one variable (Field, 2017). Welch $t$-test was used for the difference tests. Significant values were corrected using Bonferroni correction. The regression model for ID violated the linearity assumption. Thus, robust multiple regressions were performed rather than the ordinary least squares (OLS; Field \& Wilcox, 2017). The study used a between-groups design. The dependent variable was temporal planning. The predictor variables were inductive reasoning, verbal fluency, cognitive flexibility, 
Running head: Planning and Intellectual Disabilities: Support for the difference model

WM, chronological age, and activity experience. Backwards elimination based on $p$-values was used for variable selection.

Missing data Besides diagnoses, no data were missing in the MA group. In the ID group, data on the OYT was missing due to fatigue $(n=1)$, the CKTA due to technical problems $(n=5)$, the activity questionnaire $(n=4)$, the verbal fluency test $(n=3)$, and the cognitive flexibility test $(n=4)$ due to stress or nervousness. The value of the missing data was considered unrelated to the would-be values of the missing variable itself. Thus, missing data were treated as Missing At Random (MAR). All variables had less than 5\% missing data. Data were therefore imputed using the MICE-method with five imputations and then pooled using the mean value from each imputed data set (Azur, Stuart, Frangakis, \& Leaf, 2011; van Buuren \& Groothuis-Oudshoorn, 2011).

\section{Results}

The result section will first present the PCA of the planning measures, then the descriptive data and $t$-tests of the included variables followed by the correlations and regression analyses.

\section{Combining the planning variables}

Many participants reached the highest score on the planning tasks OYT and MET (OYT; $\mathrm{ID}=18 \%, \mathrm{MA}=19 \% ;$ MET; ID $=61 \%, \mathrm{MA}=61 \%)$. Therefore, the completion time was 
Running head: Planning and Intellectual Disabilities: Support for the difference model

incorporated using the Balanced Integration Score ${ }^{1}$ (BIS; Liesefeld \& Janczyk, 2019; Liesefeld, $\mathrm{Fu}, \&$ Zimmer, 2015). Due to skewness, the time variables were transformed using the natural logarithm and correlations were calculated using the Spearman correlation coefficient.

All measures correlated $\left(r_{s}>.47\right)$, except the Zoo map $\left(r_{s}<.23\right)$, which was considered to not measure the same construct of planning and was excluded in further analyses. The remaining variables were entered into a PCA with oblim rotation. The three planning variables were suitable for a PCA as indicated by a significant Bartlett test $(p<.001)$ and $\mathrm{KMO}>0.60(\mathrm{KMO}=0.68)$. The planning factor had an eigenvalue of 2.06. The loadings were: $\mathrm{OYT}=0.78, \mathrm{MET}=0.84$, $\mathrm{CKTA}=0.86$. The scores for each individual were saved using the default regression method. The underlying feature for the included tests was to organise tasks temporally (except for Zoo map), thus the new planning factor was named temporal planning.

\section{Group comparisons and descriptive statistics}

Descriptive statistics and $p$-values for the $t$-tests comparing differences in the groups can be found in Table 3. The $t$-tests showed no significant difference in any of the tasks except WM and chronological age; The MA group had higher WM scores and were younger than the ID group.

\footnotetext{
${ }^{1}$ BIS was calculated by first standardising the reaction time $\left(Z_{R T}\right)$ and the proportion correct $\left(Z_{P C}\right)$ and then subtracting the $Z_{P C}$ from the $Z_{R T}$.
} 
Running head: Planning and Intellectual Disabilities: Support for the difference model

\section{Insert Table 3 about here}

\section{Cognitive abilities and life experience to predict planning}

The two groups did not differ in temporal planning ability, $t(115.28)=0.03, p=.979$. Correlations showed that in the ID group, temporal planning correlated positively with all predictors except activity experience (non-significant) and chronological age (significant negative correlation). In the MA group, temporal planning was positively correlated with all predictors. Inter-correlations between all variables are presented in Table 4.

\section{Insert Table 4 about here}

In the regression model for MA, inductive reasoning, verbal fluency, activity experience, and chronological age were positive predictors to temporal planning, $F(4,61)=34.29, p<.001$, $R^{2}=0.44$ (Table 6 ). In the regression model for ID, WM and verbal fluency were positively related and chronological age was negatively related to temporal planning, $F(3,70)=35.54, p$ $<.001, R^{2}=0.35($ Table 5)

\section{Insert Tables 5 and 6 about here}

\section{Summary of results}

Three of the planning tests measured the same planning construct, named temporal planning. Individuals with ID did not differ from their MA peers in temporal planning performance. Different predictors explained temporal planning ability between the two groups. Verbal fluency was a predictor of planning in both groups. Chronological age was also a 
Running head: Planning and Intellectual Disabilities: Support for the difference model

predictor in both groups, but with different directions. Inductive reasoning was included for MA only, and WM was included for ID only. Activity experience predicted better planning ability in the MA group. Apart from chronological age in the ID group, all included predictors had a positive relation to temporal planning: A higher chronological age in the MA group was associated with better temporal planning whereas a lower chronological age in the ID group was associated with better temporal planning.

\section{Discussion}

\section{Life experience and temporal planning}

Life experience was able to explain temporal planning in the MA group. For the ID group, activity experience did not correlate with temporal planning nor was it included in the regression model. Even though chronological age was included in the model, the correlation was negative, suggesting that the younger the participants were the better at planning, contradicting the theory that life experience would benefit this group. The results suggest that life experience was not helping individuals in the temporal planning task.

ID students are perhaps lacking everyday practice, resulting in lower abilities with age and not gaining life experience. Individuals with ID have known meta-cognitive difficulties and difficulties transferring knowledge from one task to a new one (Nader-Grosbois, 2014). Thus, it might be beneficial to include more everyday activity training in the classrooms. However, the two groups represented two different age cohorts, and developmental changes might be more apparent in younger children than in adolescents. Thus, the different results might also be an artefact of the qualitative differences between the groups rather than being a result of the ID 
Running head: Planning and Intellectual Disabilities: Support for the difference model

itself. Younger individuals tend to have higher impulsivity and be less reflective than their older peers (Steinberg et al., 2008). This might have influenced the relationship with chronological age for the younger participants since time-to-completion was included in the planning measure. Similar results have been found in other planning tasks, like the Tower of London (Albert \& Steinberg, 2011). However, the negative effect of chronological age found in this study cannot be explained by either the difference in impulse control or time-to-completion. Rather, Albert and Steinberg (2011) found a positive correlation throughout the teenage years. Nevertheless, the speed factor could have influenced the outcome more than what was intended, due to the ceiling effect.

\section{Cognitive abilities and temporal planning}

Earlier literature has shown that verbal fluency is correlated with traditional cognitive abilities (i.e., Tower tasks; Hanes, 1996). Our results suggest that verbal fluency is also relevant for temporal planning as it predicted temporal planning in both groups. Also, as discussed above, the measure of temporal planning was based on time-to-completion. Participants with higher verbal fluency were able to finish the task quicker, resulting in a higher score on temporal planning. Thus, verbal fluency might be of extra relevance when the participants retrieve knowledge from their long-term memory or process information in their WM.

Cognitive flexibility was positively correlated with temporal planning in both groups but was not included in any of the regression models. Even though, this is contrary to some literature (e.g. Brookshire et al., 2004; Diamond, 2013; Pellicano, 2010), a review on planning in children concluded that cognitive flexibility is only necessary for planning in more complex problems where participants simultaneously manage (and switch between) a number of sub-goals 
Running head: Planning and Intellectual Disabilities: Support for the difference model

(McCormack \& Atance, 2011). Sequencing tasks temporally does not require switching among sub-goals.

Inductive reasoning predicted temporal planning in the ID group, but not in the MA group. Our finding replicated the results from Danielsson et al. (2012), where they found that MA was more related to planning in the ID group than in the MA group. However, D'Antuono et al. (2017) investigated cognitive abilities related to planning in individuals with a TD and found a very strong relationship between inductive reasoning and planning. The difference in results might be explained by Both D'Antuono et al. (2017) and Danielsson et al. (2012) using a Tower task which relies more on problem-solving, than tasks for temporal planning (Berg \& Byrd, 2002).

Literature has found WM important for planning (e.g. D’Antuono et al., 2017; Numminen et al., 2001; Unterrainer et al., 2004). WM correlated with temporal planning in both groups but was only included in the ID-model. WM might be relevant for explaining temporal planning in the MA group as well, but the effect gets masked by the inter-correlation with inductive reasoning. The strategy to solve the temporal planning tasks requires setting up a series of subgoals to achieve the final goal; This is demanding as it requires maintaining the sub-goals in the WM. The MA group might rely more on inductive reasoning since they do not have an ID. The ID group, with a reduced intellectual functioning, might be using a more WM dependent strategy. This group difference implies something qualitatively different in how individuals with ID handle temporal planning, a finding supporting the difference model. 
Running head: Planning and Intellectual Disabilities: Support for the difference model

\section{Support for the difference model of ID}

Earlier studies suggest that individuals with ID perform according to or lower than their MA in traditional planning tasks like the Tower tasks (Alloway, 2010; Danielsson et al., 2012; García-Alba et al., 2017; M. J. Van der Molen, Van Luit, Jongmans, \& Van der Molen, 2007). On a group level, this study found no differences between the groups on the planning tasks or the other cognitive tasks (besides WM). This result supports the developmental model. However, when investigating predictors associated with planning, the ID group differed qualitatively from their MA peers. Individuals with ID seem to show a different profile altogether rather than sharing a cognitive profile with individuals matched on MA. Thus, temporal planning might better be explained by the difference model of ID. The difference between the groups was captured by studying predictors for everyday planning and not only investigating group differences. This way of analysing behavioural data provides an alternative way of testing for the difference-developmental models.

\section{Limitations and future studies}

This study did not include a measure on socio-economic status, which future studies should consider. The participant's background might influence life experience and everyday planning ability. Further, the decision to incorporate time into the planning measure could have influenced the results. To avoid possible ceiling or floor effects future researchers should develop tests with a more appropriate level of difficulty for individuals with ID. Further, verbal difficulties could have affected the results as this study did not account for verbal ability or verbal WM. The study tried to minimise this risk by accompanying verbal instruction with pictures, but perhaps the results were affected if instructions still were too hard for some participants. 
Running head: Planning and Intellectual Disabilities: Support for the difference model

This study used tests that were partly modified and their reliability scores were somewhat low. However, the PCA included three of the planning measures, suggesting that the tests measured the same construct. Further, this study simulated everyday planning in an experimental setting rather than observing participants perform a planning task in their natural environment. Other variables might have been observed if investigated in a natural environment (e.g. the participant's ability to change the plan if something unexpected happened). However, the current study aimed to compare on a group-level and preferred the experimental control over absolute ecological validity.

This study included a group of participants with mixed aetiology, future studies might investigate a possible difference depending on the cause of the ID. There might be different cognitive profiles within the sample that have not been investigated.

\section{Conclusion}

Activity experience could only explain temporal planning for the MA group. Increased life experience did not help the participants with ID to plan better in everyday situations compared to younger MA-matched peers. The overall differences in the two models provide support for the difference model of ID. Chronological age was a significant predictor in both groups, but with different directions of the correlation. The ID group showed a negative correlation with chronological age and might need more everyday temporal planning training. Taken together, the results from everyday planning are more consistent with a difference model: The ID group behaved in a qualitatively different manner compared to the MA group. 
Running head: Planning and Intellectual Disabilities: Support for the difference model

\section{Practical implications}

The support for the difference model of ID implies that the methods used for individuals with a TD would perhaps not suit individuals with ID even if the method is adapted to their MA. Further, individuals with ID might not develop their everyday planning ability in later school years. Practitioners should be aware that individuals with ID might need more training in these skills throughout adolescence. To support adolescents with ID, practitioners may focus on supporting the individual's cognitive abilities rather than trusting their prior knowledge. For instance, by off-loading the individual's WM by using calendars or step-by-step schemas.

\section{Declaration of interest}

All authors declare that they have no conflicts of interest. The authors alone are responsible for the content and writing of this article. The study was funded by Stiftelsen Sävstaholm awarded to Lisa Palmqvist (ST 2016-030).

\section{References}

Albert, D., \& Steinberg, L. (2011). Age differences in strategic planning as indexed by the Tower of London. Child Development, 82(5), 1501-1517. doi:10.1111/j.1467-8624.2011.01613.x

Alloway, T. P. (2010). Working memory and executive function profiles of individuals with borderline intellectual functioning. Journal of Intellectual Disability Research, 54(5), 448-456. doi:10.1111/j.1365-2788.2010.01281.x 
Running head: Planning and Intellectual Disabilities: Support for the difference model

Ardila, A. (2008). On the evolutionary origins of executive functions. Brain and Cognition, 68(1), 92-99. doi:10.1016/j.bandc.2008.03.003

Arvidsson, P., \& Granlund, M. (2018). The relationship between intelligence quotient and aspects of everyday functioning and participation for people who have mild and borderline intellectual disabilities. Journal of Applied Research in Intellectual Disabilities, 31(1), e68-e78. doi:10.1111/jar.12314

Aust, F., \& Barth, M. (2018). papaja: Create APA manuscripts with R Markdown. Retrieved from https://github.com/crsh/papaja

Azur, M. J., Stuart, E. A., Frangakis, C., \& Leaf, P. J. (2011). Multiple imputation by chained equations: what is it and how does it work? International Journal of Methods in Psychiatric Research, 20(1), 40-9. doi:10.1002/mpr.329

Baldo, J. V., Shimamura, A. P., Delis, D. C., Kramer, J. H., \& Kaplan, E. (2001). Verbal and design fluency in patients with frontal lobe lesions. Journal of the International Neuropsychological Society, 7(5), 586-596. doi:10.1017/S1355617701755063

Bennett-Gates, D., \& Zigler, E. (1998). Resolving the developmental-difference debate: An evaluation of the triarchic and systems theory models. In Handbook of mental retardation and development (pp. 115-131).

Berg, W. K., \& Byrd, D. L. (2002). The Tower of London spatial problem-solving task: Enhancing clinical and research implementation. Journal of Clinical and Experimental Neuropsychology, 24(5), 586-604. doi:10.1076/jcen.24.5.586.1006 
Running head: Planning and Intellectual Disabilities: Support for the difference model

Brookshire, B., Levin, H., Song, J., \& Zhang, L. (2004). Components of executive function in typically developing and head-injured children. Developmental Neuropsychology, 25(1), 61-83. doi:10.1207/s15326942dn2501\&2_5

Burgess, P., Alderman, N., Evans, J., Emslie, H., \& Wilson, B. A. (1998). The ecological validity of tests of executive function. Journal of the International Neuropsychological Society, 4(06), 547-558. doi:10.1017/S1355617798466037

Burgess, P., Alderman, N., Forbes, C., Costello, A., Coates, L., Dawson, D. R., ... Channon, S. (2006). The case for the development and use of "ecologically valid" measures of executive function in experimental and clinical neuropsychology. Journal of the International Neuropsychological Society, 12(2), 194-209. doi:10.1017/S1355617706060310

Chalmers, D., \& Lawrence, J. A. (1993). Investigating the effects of planning aids on adults' and adolescents' organisation of a complex task. International Journal of Behavioral Development, 16(2), 191-214. doi:10.1177/016502549301600206

Chevignard, M., Catroppa, C., Galvin, J., \& Anderson, V. (2010). Development and evaluation of an ecological task to assess executive functioning post childhood TBI: The Children's Cooking Task. Brain Impairment, 11(2), 125-143. doi:10.1375/brim.11.2.125

Danielsson, H., Henry, L. A., Messer, D., \& Rönnberg, J. (2012). Strengths and weaknesses in executive functioning in children with intellectual disability. Research in Developmental Disabilities, 33(2), 600-607. doi:10.1016/j.ridd.2011.11.004 
Running head: Planning and Intellectual Disabilities: Support for the difference model

Danielsson, H., Henry, L. A., Rönnberg, J., \& Nilsson, L.-G. (2010). Executive functions in individuals with intellectual disability. Research in Developmental Disabilities, 6(6), 1299-1304. doi:10.1016/j.ridd.2010.07.012

Diamond, A. (2013). Executive functions. Annual Review of Psychology, 64, 135-68. doi:10.1146/annurev-psych-113011-143750

D’Antuono, G., La Torre, F. R., Marin, D., Antonucci, G., Piccardi, L., \& Guariglia, C. (2017). Role of working memory, inhibition, and fluid intelligence in the performance of the Tower of London task. Applied Neuropsychology:Adult, 24(6), 548-558. doi:10.1080/23279095.2016.1225071

Emslie, H., Wilson, C., Burden, V., Nimmo-Smith, I., \& Wilson, B. (2003). Behavioural assessment of the dysexecutive syndrome for children (BADS-C). London, UK: Harcourt Assessment/The Psychological Corporation.

Facon, B., Magis, D., \& Belmont, J. M. (2011). Beyond matching on the mean in developmental disabilities research. Research in Developmental Disabilities, 32(6), 2134-2147. doi:10.1016/j.ridd.2011.07.029

Field, A. P. (2017). Discovering statistics using IBM SPSS Statistics (5th ed., p. 1104). SAGE.

Field, A. P., \& Wilcox, R. R. (2017). Robust statistical methods: A primer for clinical psychology and experimental psychopathology researchers. Behaviour Research and Therapy, 98, 19-38. doi:10.1016/j.brat.2017.05.013 
Running head: Planning and Intellectual Disabilities: Support for the difference model

Fox, J., \& Weisberg, S. (2011). An R companion to applied regression (2nd ed.). Thousand Oaks CA: Sage.

Friedman, S. L., \& Scholnick, E. K. (2014). An evolving "blueprint" for planning: psychological requirements, task characteristics, and social-cultural influences. In The developmental psychology of planning (pp. 3-22). Psychology Press.

García-Alba, J., Esteba-Castillo, S., Castellanos López, M. Á., Rodríguez Hidalgo, E., Ribas Vidal, N., Moldenhauer Díaz, F., \& Novell-Alsina, R. (2017). Validation and normalization of the Tower of London-Drexel University Test $2^{\text {nd }}$ Edition in an adult population with intellectual disability. The Spanish Journal of Psychology, 20, E32. doi:10.1017/sjp.2017.30

Hanes, K. (1996). A brief assessment of executive control dysfunction: Discriminant validity and homogeneity of planning, set shift, and fluency measures. Archives of Clinical Neuropsychology, 11(3), 185-191. doi:10.1016/0887-6177(95)00034-8

Hayes-Roth, B., \& Hayes-Roth, F. (1979). A cognitive model of planning. Cognitive Science, 3(4), 275-310. doi:10.1016/S0364-0213(79)80010-5

Jurado, M. B., \& Rosselli, M. (2007). The elusive nature of executive functions: A review of our current understanding. Neuropsychology Review, 17(3), 213-233. doi:10.1007/s11065007-9040-z 
Running head: Planning and Intellectual Disabilities: Support for the difference model

Kliegel, M., Martin, M., McDaniel, M. A., \& Phillips, L. H. (2007). Adult age differences in errand planning: The role of task familiarity and cognitive resources. Experimental Aging Research, 33(2), 145-161. doi:10.1080/03610730601177395

Lanfranchi, S., Jerman, O., Dal Pont, E., Alberti, A., \& Vianello, R. (2010). Executive function in adolescents with Down Syndrome. Journal of Intellectual Disability Research, 54(4), 308-319. doi:10.1111/j.1365-2788.2010.01262.x

Levine, B., Robertson, I. H., Clare, L., Carter, G., Hong, J., Wilson, B. A., ... Stuss, D. T. (2000). Rehabilitation of executive functioning: An experimental-clinical validation of Goal Management Training. Journal of the International Neuropsychological Society, 6(3), 299-312. doi:10.1017/S1355617700633052

Liesefeld, H. R., \& Janczyk, M. (2019). Combining speed and accuracy to control for speedaccuracy trade-offs(?). Behavior Research Methods, 51(1), 40-60. doi:10.3758/s13428018-1076-X

Liesefeld, H. R., Fu, X., \& Zimmer, H. D. (2015). Fast and careless or careful and slow? Apparent holistic processing in mental rotation is explained by speed-accuracy trade-offs. Journal of Experimental Psychology: Learning Memory and Cognition, 41(4), 11401151. doi:10.1037/xlm0000081

Masson, J. D., Dagnan, D., \& Evans, J. (2010). Adaptation and validation of the Tower of London test of planning and problem solving in people with intellectual disabilities. Journal of Intellectual Disability Research, 54(5), 457-467. doi:10.1111/j.13652788.2010.01280.x 
Running head: Planning and Intellectual Disabilities: Support for the difference model

McCormack, T., \& Atance, C. M. (2011, March). Planning in young children: A review and synthesis. Academic Press. doi:10.1016/j.dr.2011.02.002

Miyake, A., Friedman, N. P., Emerson, M. J., Witzki, A. H., Howerter, A., \& Wager, T. D. (2000). The unity and diversity of executive functions and their contributions to complex “frontal lobe" tasks: A latent variable analysis. Cognitive Psychology, 41(1), 49-100. doi:10.1006/cogp.1999.0734

Mueller, S. T., \& Piper, B. J. (2014). The Psychology Experiment Building Language (PEBL) [Measurement instrument]. Journal of Neuroscience Methods, 222, 250-259. doi:10.1016/j.jneumeth.2013.10.024

Nader-Grosbois, N. (2014). Self-perception, self-regulation and metacognition in adolescents with intellectual disability. Research in Developmental Disabilities, 35(6), 1334-1348. doi:10.1016/J.RIDD.2014.03.033

Numminen, H., Lehto, J. E., \& Ruoppila, I. (2001). Tower of Hanoi and working memory in adult persons with intellectual disability. Research in Developmental Disabilities, 22(5), 373-387. doi:10.1016/S0891-4222(01)00078-6

Pellicano, E. (2010). The development of core cognitive skills in autism: A 3-year prospective study. Child Development, 81(5), 1400-1416. doi:10.1111/j.1467-8624.2010.01481.x

Pennington, B. F., \& Ozonoff, S. (1996). Executive functions and developmental psychopathology. Journal of Child Psychology and Psychiatry, 37(1), 51-87. doi:10.1111/j.1469-7610.1996.tb01380.x 
Running head: Planning and Intellectual Disabilities: Support for the difference model

Pennington, B. F., Moon, J., Edgin, J., Stedron, J., \& Nadel, L. (2003). The neuropsychology of Down Syndrome: Evidence for hippocampal dysfunction. Child Development, 74(1), 7593. doi:10.1111/1467-8624.00522

Raven, J. (2003). Raven Progressive Matrices. In Handbook of nonverbal assessment (pp. 223237). Boston, MA: Springer US. doi:10.1007/978-1-4615-0153-4_11

Revelle, W. (2018). Psych: Procedures for psychological, psychometric, and personality research. Evanston, Illinois: Northwestern University. Retrieved from https://CRAN.Rproject.org/package=psych

Rowe, J., Lavender, A., \& Turk, V. (2006). Cognitive executive function in Down's syndrome. British Journal of Clinical Psychology, 45(1), 5-17. doi:10.1348/014466505X29594

Scholnick, E. K., \& Friedman, S. L. (1993). Planning in context: Developmental and situational considerations. International Journal of Behavioral Development, 16(2), 145-167. doi:10.1177/016502549301600204

Schuchardt, K., Gebhardt, M., \& Mähler, C. (2010). Working memory functions in children with different degrees of intellectual disability. Journal of Intellectual Disability Research, 54(4), 346-353. doi:10.1111/j.1365-2788.2010.01265.x

Shallice, T. (1982). Specific impairments of planning. Philosophical Transactions of the Royal Society B: Biological Sciences, 298(1089), 199-209. doi:10.1098/rstb.1982.0082

Shallice, T., \& Burgess, P. (1991). Deficits in strategy application following frontal lobe damage in man. Brain, 114(2), 727-741. doi:10.1093/brain/114.2.727 
Running head: Planning and Intellectual Disabilities: Support for the difference model

Simon, H. A. (1975). The functional equivalence of problem solving skills. Cognitive Psychology, 7(2), 268-288. doi:10.1016/0010-0285(75)90012-2

Steinberg, L., Albert, D., Cauffman, E., Banich, M., Graham, S., \& Woolard, J. (2008). Age differences in sensation seeking and impulsivity as indexed by behavior and self-report: Evidence for a dual systems model. Developmental Psychology, 44(6), 1764-1778. doi:10.1037/a0012955

Steverson, T., Adlam, A.-L. R., \& Langdon, P. E. (2017). Development and validation of a modified multiple errands test for adults with intellectual disabilities. Journal of Applied Research in Intellectual Disabilities, 30(2), 255-268. doi:10.1111/jar.12236

Todorov, V., \& Filzmoser, P. (2009). An object-oriented framework for robust multivariate analysis. Journal of Statistical Software, 32(3), 1-47.

Unterrainer, J. M., Rahm, B., Kaller, C. P., Leonhart, R., Quiske, K., Hoppe-Seyler, K., ... Halsband, U. (2004). Planning abilities and the Tower of London: Is this task measuring a discrete cognitive function? Journal of Clinical and Experimental Neuropsychology, 26(6), 846-856. doi:10.1080/13803390490509574

van Buuren, S., \& Groothuis-Oudshoorn, K. (2011). mice: Multivariate imputation by chained equations in r. Journal of Statistical Software, 45(3), 1-67

Van der Molen, M. J., Van Luit, J. E. H., Jongmans, M. J., \& Van der Molen, M. W. (2007). Verbal working memory in children with mild intellectual disabilities. Journal of Intellectual Disability Research, 51(2), 162-169. doi:10.1111/j.1365-2788.2006.00863.x 
Running head: Planning and Intellectual Disabilities: Support for the difference model

Wickham, H., François, R., Henry, L., \& Müller, K. (2019). Dplyr: A grammar of data manipulation. Retrieved from https://CRAN.R-project.org/package=dplyr

Willner, P., Bailey, R., Parry, R., \& Dymond, S. (2010). Evaluation of executive functioning in people with intellectual disabilities. Journal of Intellectual Disability Research, 54(4), 366-379. doi:10.1111/j.1365-2788.2010.01249.x

World Health Organization. (2001). The international classification of functioning, disability and health: ICF (p. 237). Geneva, Switzerland: World Health Organization. doi:10.1097/01.pep.0000245823.21888.71 
Running head: Planning and Intellectual Disabilities: Support for the difference model

Table 1. Diagnoses in the ID group

Diagnoses $n$

Mild intellectual disability only 30

Down Syndrome $\quad 7$

Williams Syndrome $\quad 1$

Other biological cause 3

Mild intellectual disability and Autism spectrum disorder 9

Mild intellectual disability and Attention deficit hyperactivity disorder 7

Mild intellectual disability and dyslexia 2

Mild intellectual disability and language disorder 2

$\begin{array}{ll}\text { More than one additional diagnosis } & 10\end{array}$

$\begin{array}{ll}\text { Total } & 71\end{array}$ 
Running head: Planning and Intellectual Disabilities: Support for the difference model

Table 2. Detailed descriptions of the included tests. The first four captured everyday planning. The next four cognitive abilities. Last, a questionnaire capturing life experience was included. None of the included task had a time constraint.

Planning task 1: Order-your-task

The Order-your-task (OYT) test was developed for this project to assess the participant's ability to temporally organise a task into sub-tasks (e.g. for the task "Eat a slice of toast", identify the order of the sub-tasks: 1. Toast bread, 2. Spread butter onto toast, and 3. Eat toast. The participant received ten tasks yielding one point per correct answer (maximum score $=10$ ). The dependent variable was the total correct tasks combined with the time to completion.

Planning task 2: Multiple errand task

The multiple errand task (MET; Shallice \& Burgess, 1991) originally captured difficulties with everyday tasks in individuals with acquired brain injuries and have been adapted for individuals with ID (Steverson et al., 2017). In the original task, the participant is asked to execute a set of errands in a shopping mall. The present study aimed to investigate the formulation rather than the execution of the plan, thus, the task was modified into a pen-andpaper task. The participants were given a list of seven tasks to perform while following a series of rules. The setting was a school environment, and the tasks included everyday school activities such as, "draw a sun", "fetching a pen", "playing football", and "finding out what's for lunch". The rules included finding the appropriate room to visit (e.g. go to the school yard for playing football), not to enter the same room twice, and doing the task in the appropriate order (e.g. fetching the pen before drawing the sun). One point was given per correct matched pair of rooms and tasks, 1.25 points were given if the tasks were performed in an appropriate order, and one minus was given if the participant entered the same room twice (maximum score $=8.25$ ). The dependent variable was the total correct tasks combined with the time to completion.

Planning task 3: Children's Kitchen Task Assessment

The Children's Kitchen Task Assessment (CKTA) was developed by Chevignard et al. (2010). The CKTA was modified to a pen-and-paper task. The participant was asked to identify the correct steps in a recipe (maximum score $=8$ ). The dependent variable was the total correct tasks combined with the time to completion. 
Running head: Planning and Intellectual Disabilities: Support for the difference model

Planning task 4: Zoo map

The Zoo map 1 (from here on referred to as Zoo Map) is a sub-test in the test battery BADS-C (Emslie et al., 2003). The Zoo map assesses the participant's own ability to identify and arrange sub-goals to achieve a goal. The participant is asked to visit eight places in a zoo (e.g. the restaurant or the bears) using a paper map of the zoo. The participant may only use certain roads once and must start and end in specified places. The participant is prompted to draw a line with a pen showing what places they visited and in which order. The participant got one score per correct visited animal. The score was then deduced by one each time the participant: used a forbidden path more than once, deviated from the path, failed to draw a continuous line, or made an inappropriate visit (maximum score $=8$ ). The dependent variable was the total correct tasks combined with the time to completion.

Working memory

Executively loaded visuo-spatial working memory capacity (WM) was measured using a backwards Corsi span on the software Pebl (Mueller \& Piper, 2014)). Corsi span is a spatial task used to assess the spatial WM. The test consisted of a grid of $3 \times 3$ squares. The squares lit up, one by one in a sequence, and the participants were asked to remember the correct reversed order. The participant was then asked to click on the squares in the correct pattern using a computer mouse. The test started with a sequence of two squares and increased by one square every second sequence. The test ended when a participant made two errors in a row. The participant was given a test trial consisting of three supervised 3-item-spans. The participant was verbally reminded that the task was to remember the sequence backwards to avoid floor effects due to forgetting instructions. The score was calculated by taking the minimum list length, adding the total number correct, and then dividing the number of lists at each length. This results in the participant's mean span which corresponds to the participant's working memory capacity. The dependent variable was the participant's working memory span.

Inductive reasoning

The participants' non-verbal inductive reasoning was measured using Raven's Coloured Progressive Matrices (Raven, 2003). The total number of correct was calculated (maximum score $=36$ ). The dependent variable was the total score.

Cognitive flexibility 
Running head: Planning and Intellectual Disabilities: Support for the difference model

Playing Cards in BADS-C was used for assessing cognitive flexibility (Emslie et al., 2003). The participant was asked to reply "yes" or "no" according to a pre-specified rule. The test was performed two times, with different rules. If the participants successfully switched to the new rule (i.e. no rule breaks) they were given a score of 1 and if they failed (i.e. one or more rule breaks) the participant was given a 0 . That is, the dependent variable was binary ( 1 or 0$)$.

Verbal fluency

The semantic category fluency task was used to measure verbal fluency (Baldo et al., 2001). The participants were asked to name as many items as possible in two given categories (animals and boy names) for $2 * 60$ seconds. The category test was used rather than the letter fluency task (e.g. FAS) to reduce the influence on the participants reading skills. The total number of uniquely named items was calculated and used as the dependent variable.

Activity Experience

Activity experience was measured using a short version of a questionnaire developed by Arvidsson and Granlund (2018) for individuals with ID. The questionnaire was originally developed to measure the individual's participation in society, only the component that captured how often an individual did a certain activity was included (e.g. "How often do you take the bus to school?", "How often do you shop in stores?", and "How often do you visit a café/restaurant"). The questionnaire is a structured interview where the participant rate how often they perform everyday activities ( $2=$ often, $1=$ sometimes, $0=$ seldom $/$ never $)$. The questionnaire included 22 items representing a selection of categories from the ICF domains of the activity/participation component (World Health Organization, 2001). The original questionnaire did not include any questions regarding how often the participant baked. Since one of the planning measured used recipes, this question was added to the questionnaire. The dependent variable of the activity experience was the sum of the scores on the 23 items (maximum 46). 
Running head: Planning and Intellectual Disabilities: Support for the difference model

Table 3. Descriptive statistics of included variables, p-values were calculated with $t$-tests using Bonferroni correction for significant p-values.

\begin{tabular}{lcllllll} 
& \multicolumn{1}{c}{ Intellectual disability } & \multicolumn{3}{c}{ Mental-age matched } \\
\hline & $M$ & $S D$ & $($ Range $)$ & $M$ & $S D$ & $($ Range $)$ & $p$ \\
\hline Chronological age & 18.1 & 1.30 & $(16,21)$ & 7.8 & 0.73 & $(6,9)$ & $<.001$ \\
Inductive reasoning & 25 & 5.4 & $(15,35)$ & 25 & 5.0 & $(15,35)$ & .555 \\
Temporal planning & 0.039 & 1.11 & $(-3,2)$ & 0.034 & 0.78 & $(-1,2)$ & .979 \\
Verbal fluency & 38 & 12.1 & $(18,74)$ & 37 & 8.7 & $(20,55)$ & .482 \\
Cognitive flexibility & 0.30 & 0.46 & $(0,1)$ & 0.37 & 0.49 & $(0,1)$ & .388 \\
Working Memory & 5.9 & 2.3 & $(1,10)$ & 7.0 & 1.8 & $(2,10)$ & .008 \\
Activity Experience & 28 & 5.3 & $(17,37)$ & 25 & 4.5 & $(15,35)$ & .119
\end{tabular}


Running head: Planning and Intellectual Disabilities: Support for the difference model

Table 4. Inter-correlations between temporal planning and the predictors for the ID group (upper right triangle), and MA group (lower left triangle).

\begin{tabular}{llllllll} 
& 1. & 2. & 3. & 4. & 5. & 6. & 7. \\
\hline 1. Temporal planning & & $.32^{* *}$ & $.30^{*}$ & $.32^{* *}$ & $.40^{* *}$ & $.23 \dagger$ & $-.40^{* *}$ \\
2. Inductive reasoning & $.41^{* *}$ & & $.20 \dagger$ & $.38^{* *}$ & $.56^{* *}$ & .02 & $-.31^{* *}$ \\
3. Verbal fluency & $.50^{* *}$ & .13 & & $.20 \dagger$ & $.26^{*}$ & $.32^{* *}$ & -.19 \\
4. Cognitive flexibility & $.29^{*}$ & $.25 \dagger$ & .07 & & $.25^{*}$ & .01 & -.19 \\
5. Working memory & $.33^{* *}$ & $.37^{* *}$ & $.33^{* *}$ & .11 & & .17 & $-.25^{*}$ \\
6. Activity Experience & $.41^{* *}$ & .07 & $.27^{*}$ & .17 & .06 & & -.07 \\
7. Chronological age & $.46^{* *}$ & $.25 \dagger$ & $.28^{*}$ & $.22 \dagger$ & $.30^{*}$ & $.34^{* *}$ & \\
\hline
\end{tabular}

Note. $* * *=p<.001, * *=p<.01, *=p<.05, \dagger=p<.10$. 
Running head: Planning and Intellectual Disabilities: Support for the difference model

Table 5. Coefficients for the regression for adolescents with

Intellectual Disabilities

\begin{tabular}{lllll}
\hline & $\beta$ & $S E$ & $t(67)$ & $p$ \\
Working memory & 0.28 & 0.1 & 2.62 & .011 \\
Verbal fluency & 0.25 & 0.1 & 2.38 & .020 \\
Chronological age & -0.34 & 0.1 & -3.23 & .002 \\
\hline
\end{tabular}

Table 6. Coefficients for the regression for the Mental-age matched children

\begin{tabular}{lllll}
\hline & $\beta$ & $S E$ & $t(57)$ & $p$ \\
Inductive reasoning & 0.27 & 0.11 & 2.51 & .015 \\
Verbal fluency & 0.33 & 0.11 & 2.93 & .005 \\
Activity experience & 0.19 & 0.09 & 2.05 & .045 \\
Chronological age & 0.24 & 0.11 & 2.21 & .031 \\
\hline
\end{tabular}

